\title{
Structural Optimization of Cantilever Beam in Conjunction with Dynamic Analysis
}

\author{
Behzad Ahmed Zai - Furqan Ahmad - Chang Yeol Lee \\ Tae-Ok Kim** ${ }^{\dagger}$ Myung Kyun Park \\ Department of Mechanical Engineering, Myongji University, Yongin 449-728, Korea \\ "Department of Chemical Engineering, Myongji University, Yongin 449-728, Korea \\ (Received July 20, 2011; Revised October 28, 2011; Accepted October 28, 2011)
}

\begin{abstract}
In this paper, an analytical model of a cantilever beam having a midpoint load is considered for structural optimization and design. This involves creation of the geometry through a parametric study of all design variables. For this purpose, the optimization of the cantilever beam was elaborated in order to find the optimum geometry which minimizes its volume eventually for minimum weight by FEM (finite element method) analysis. Such geometry can be obtained by different combinations of width and height, so that the beam may have the same cross-sectional area, yet different dynamic behavior. So for optimum safe design, besides minimum volume it should have minimum vibration as well. In order to predict vibration, different dynamic analyses were performed simultaneously to identify the resonant frequencies and mode shapes belonging to the lowest three modes of vibration. Next, by introducing damping effects, the tip displacement and bending stress at the fixed end was evaluated under dynamic loads of varying frequency. Investigation of the results clearly shows that only structural analysis is not enough to predict the optimum values of dimension for safe design it must be aided by dynamic analysis as well.
\end{abstract}

Key words : optimization, natural frequencies, state space form, dynamic analysis, resonance

\section{INTRODUCTION}

As the structures become more advanced, our requirements more ambitious, and operating environments more uncertain, it is increasingly important to rigorously account for variability in the design process by considering each and every aspect of design, either static or dynamic[1,2]. This paper has developed such a methodology for three-dimensional reliability-based optimization of cantilever beam by defining design, state and objective variables[3,4]. Once the optimization is done, one may start structural frequency response testing or modal analysis for prediction of resonance and tip vibration[5]. The whole scheme follows the procedure described in Fig. 1.

The optimization program, based on the method presented in this paper, runs iteratively in the steps described in Fig. 1[6,7]. First of all, a finite element model was formed for the initial rough dimensions.

${ }^{\dagger}$ Corresponding author:pmk@mju.ac.kr
Then a finite element solver solves for state variables, such as working stress, whereas the local von Mises stress distribution was used as information to adjust the

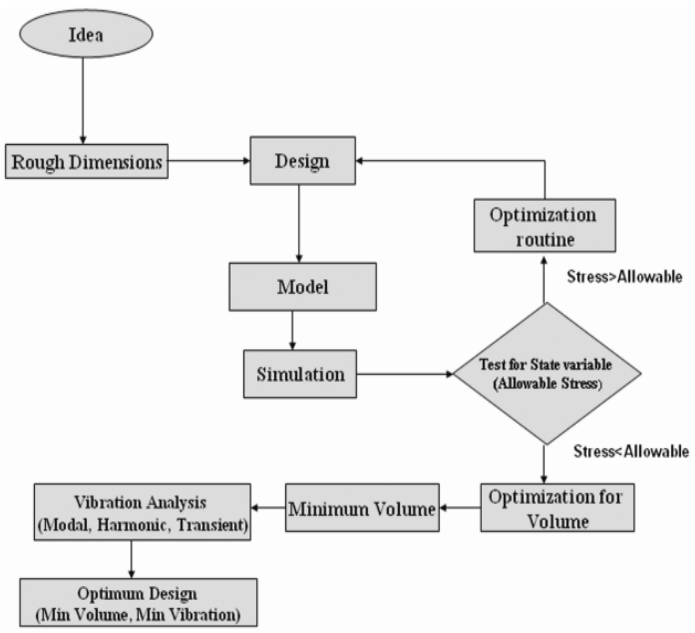

Fig. 1. Optimization procedure. 
dimensions until working stress is less than allowable stress. After the adjustment, a new finite element model was created and the process repeated[8]; in this phase, the attempt was made to reduce volume without exceeding the stress, which is called optimization for volume.

After getting the minimum volume, which is one of the main objectives, we took different combinations of width and height which produced the same volume and performed the vibration analysis for each set to find the best dimensional set of minimum volume as well as minimum vibration.

\section{II . OPTIMIZATION APPROACH}

The purpose of this optimization is to minimize the weight of the beam without exceeding the allowable stress[3,4]. An aluminum cantilever beam was taken having module of elasticity, $E=71 \mathrm{GPa}$, density, $\rho=$ $2770 \mathrm{~kg} / \mathrm{m}^{3}$ and Poisson's ratio, $v=0.33$. The beam was subjected to a force $(F)$ of $1000 \mathrm{~N}$ which is applied at the middle of the beam in the direction as shown in Fig. 2. Where $H, L$ and $W$ are the height, length and width of the beam, respectively. It is necessary to find the cross-sectional dimensions of the beam in order to minimize the weight. However, it was assumed that the width and the height of the beam cannot be smaller than $10 \mathrm{~mm}$ and for the whole analysis, the length of the beam was fixed at $1000 \mathrm{~mm}$. As well, the allowable stress caused by static load, $F$ in the beam structure cannot exceed $100 \mathrm{MPa}$. We start optimization with some arbitrary values of height and width[2,5,6].

According to the beam theory[8], the major contribution to the element's von Mises stress comes from bending stress, although transverse shear stress exists.

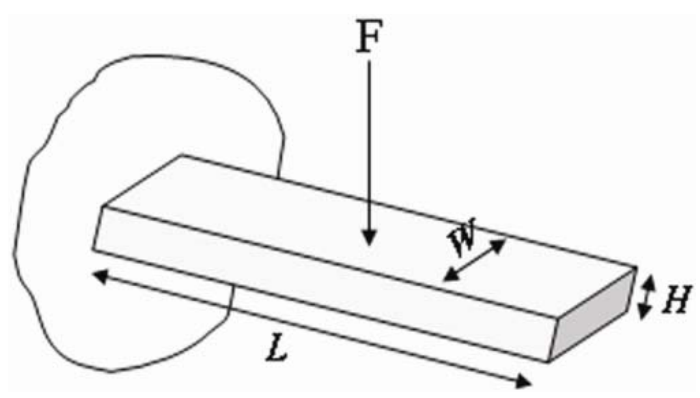

Fig. 2. Cantilever beam used for analysis.
The bending stress at any element of beam is a function of bending moment $(M)$, the transverse location from the neutral axis (y), and the beam's sectional moment of inertia $(I)$ as shown in Eq. (1).

$$
\sigma=\frac{M y}{I}
$$

Where, $y=H / 2$ and $I=W H^{3} / 12$.

FEM (finite element method) analysis needs to know which variables are critical for optimization by defining design[3,4], state and objective variables. So in this analysis, width and height were defined as design variables which are independent variables and directly affect the solution of the problem. State variable is the first dependent variable, and so in this analysis maximum stress was defined as a state variable which acts as a constraint for the problem. Since the primary objective is to reduce volume without exceeding the allowable stress in the beam, volume was defined as an objective variable. This is the one variable in the optimization which needs to be minimized. Starting with the rough values of height and width of $25 \mathrm{~mm}$, we have higher than the allowable stress. Hence, this result is not feasible, and iteration is continued until optimized results are achieved as shown in Fig. 3, Fig. 4, and Fig. 5.

The $25^{\text {th }}$ iteration set is the optimum result having a volume of $600100 \mathrm{~mm}^{3}$. Modal analysis was done by using this optimum as initial point. Method of "First order" was selected for optimization which quickly

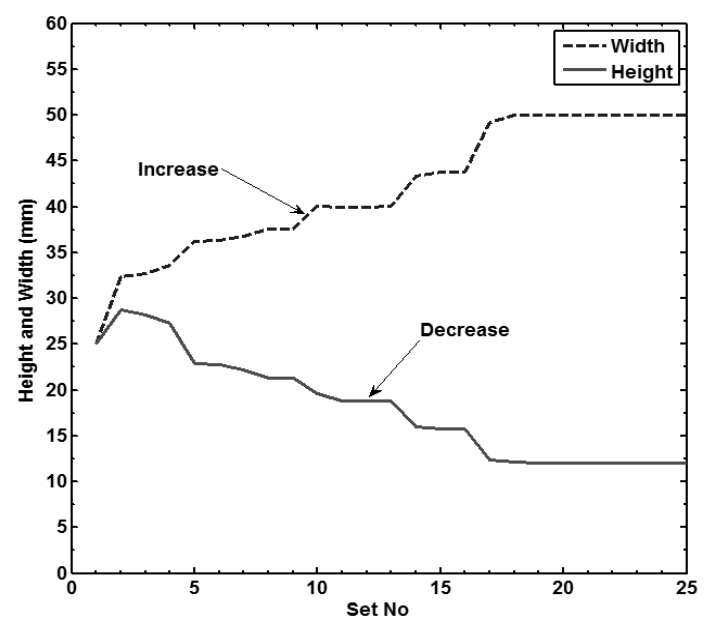

Fig. 3. Height and width by optimization. 


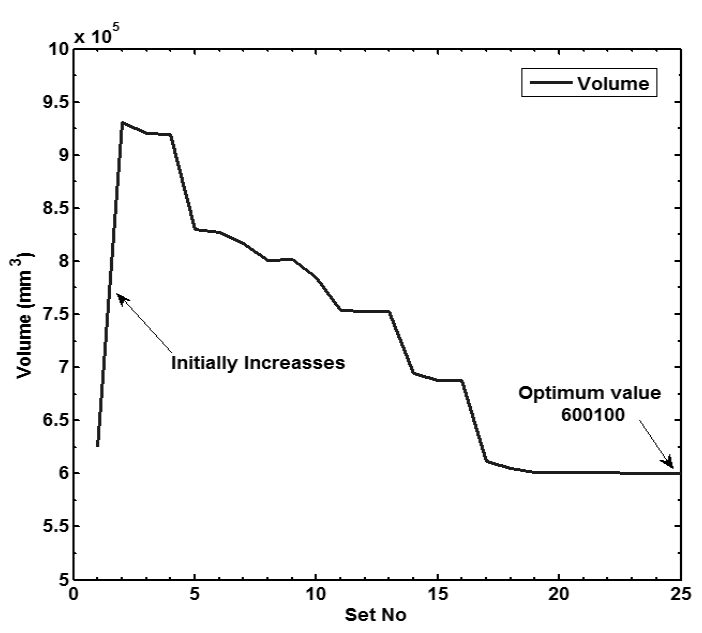

Fig. 4. Volume by optimization.

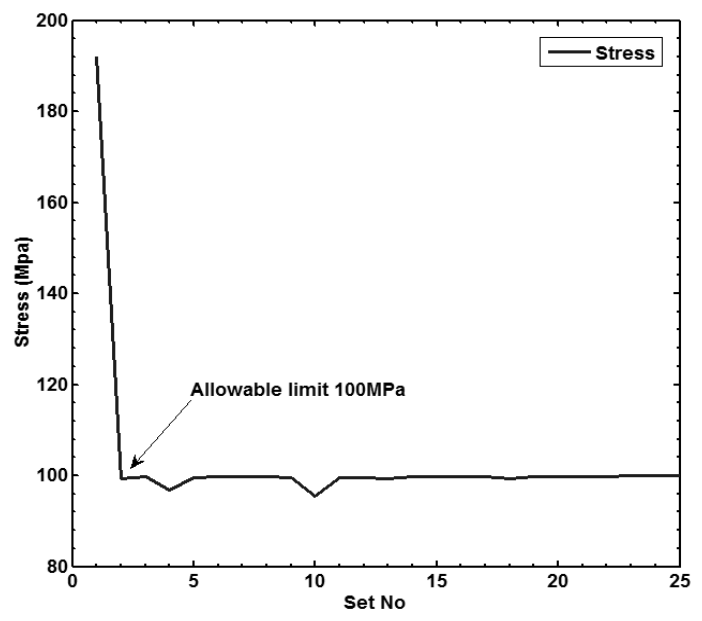

Fig. 5. Working stress during optimization. converged the solution to optimum results[3,4]. Starting from the rough values, initially both the width and the height increase to bring down the stress level that is allowable, and therefore decrease the volume to reduce the weight without exceeding the stress in the beam as shown in Fig. 4 and Fig. 5. As mentioned earlier, at first optimization stresses were brought down, and then volume was reduced without exceeding the stress as shown in Fig. 5. During optimization, the stress was within the range of $95 \mathrm{MPa}$ to $100 \mathrm{MPa}$.

\section{DYNAMIC ANALYSIS}

\subsection{Dimensions for dynamic analysis}

By executing the 25th iteration, we have an optimum volume of $600100 \mathrm{~mm}^{3}$ with height $50 \mathrm{~mm}$, width $12.002 \mathrm{~mm}$, but this volume can also be obtained within $\pm 1 \%$ by using the dimensions listed in Table 1 . The $3^{\text {rd }}$ set number is the optimum value obtained through FEM analysis; then by changing the dimension arbitrarily by keeping the same volume within $\pm 1 \%$ as listed in Table 1, the dynamic analysis was done to obtain the optimum dimension with minimum vibration. Although the $4^{\text {th }}$ and $5^{\text {th }}$ sets produce the optimum volume, they cannot be used for analysis because they are infeasible as far as allowable stress is concerned. The maximum stress was obtained from stress analysis using the geometry in Table 1 .

\subsection{Modal analysis}

In general, modal analysis of structure should be initially performed to provide information about the structure's dynamic behavior. Modal analysis can be performed by using a commercial FEM package like

Table 1. Dimensions selected for further dynamic analysis

\begin{tabular}{|c|c|c|c|c|c|}
\hline Set No. & Height $(\mathrm{mm})$ & Width $(\mathrm{mm})$ & Volume $\left(\mathrm{mm}^{3}\right)$ & Max. stress $(\mathrm{MPa})$ & Status \\
\hline 1 & 10 & 60 & 600000 & 83 & $\mathrm{~F}^{\mathrm{a})}$ \\
\hline 2 & 11 & 55 & 605000 & 91 & $\mathrm{~F}$ \\
\hline 3 & 12.002 & 50 & 600100 & 100 & $\mathrm{~F}$ \\
\hline 4 & 13 & 46 & 598000 & 109 & $\mathrm{~N}^{\mathrm{b})}$ \\
\hline 5 & 14 & 43 & 602000 & 108 & $\mathrm{~N}$ \\
\hline
\end{tabular}

a) F: Feasible

b) $\mathrm{N}$ : Infeasible 
ANSYS. The FEM results are compared with theoretical results and are shown in Table 2.

\subsubsection{Theory}

Transverse or flexural motion of a homogeneous, isotropic, and linear elastic beam without shear or rotary inertia effect is described by the well known Bernoulli-Euler Equation.

$$
-\frac{\partial^{2}}{\partial x^{2}}\left(E I \frac{\partial^{2} w}{\partial x^{2}}\right)=\rho A \frac{\partial^{2} W}{\partial t^{2}}
$$

Where, $w$ is transverse displacement of the centroidal axis of beam and $x$ is distance from the beam If we solve this partial differential equation with proper boundary conditions, the mode shapes and frequencies for the first three modes of the cantilever beam can be obtained; the results are shown in Fig. 6[9].

\subsubsection{FEM model}

Three-dimensional elements were used to generate the cantilever beam model and the FEM model for set number 3 is shown in Fig. 7. 100 brick elements with 8 nodes were used for set number 3 .

\subsection{Transient analysis}

It is difficult to decide on the basis of natural frequencies which set number is a final optimum for the design, because there is not a large difference in the natural frequencies. So the tip displacements for each set number were evaluated by performing the transient analysis, at which a time a varying load was to be imposed on the middle of the beam in the y direction of value of $-1000 \mathrm{~N}$ as shown in Fig. 8 .

For this type of analysis the inertia or damping effect of structure are included, and the damping factor is $1 \%$ of the critical, i.e. $\zeta=0.01$. The Fig. 9 shows the vibration displacement at the cantilever beam tip for all three sets of dimensions.
Both set number 1 and 2 have larger vibration displacements than set number 3; however, set number 1 gives less bending stress than set number 3 as compared
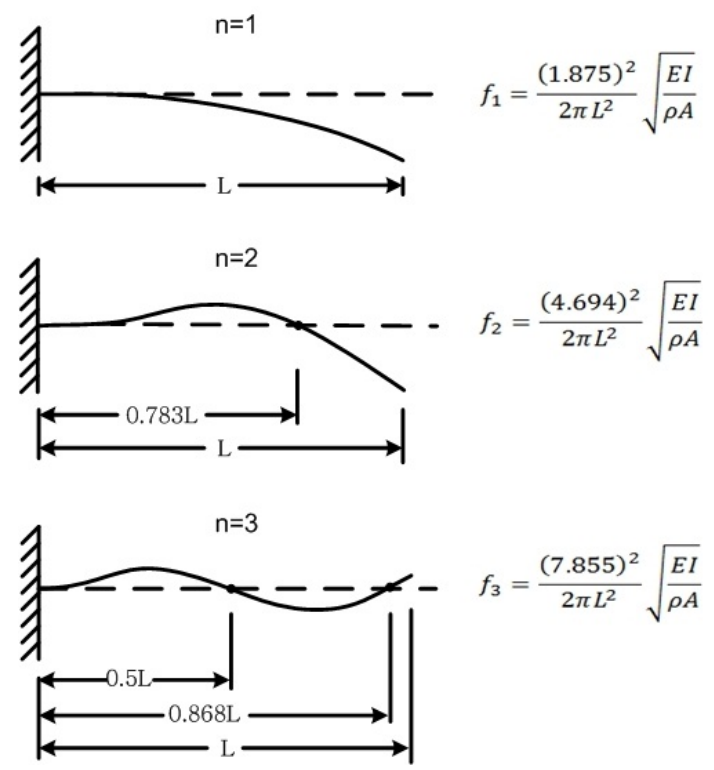

Fig. 6. Mode shapes and natural frequencies for the first three modes of flexural vibration of the cantilever beam[9].

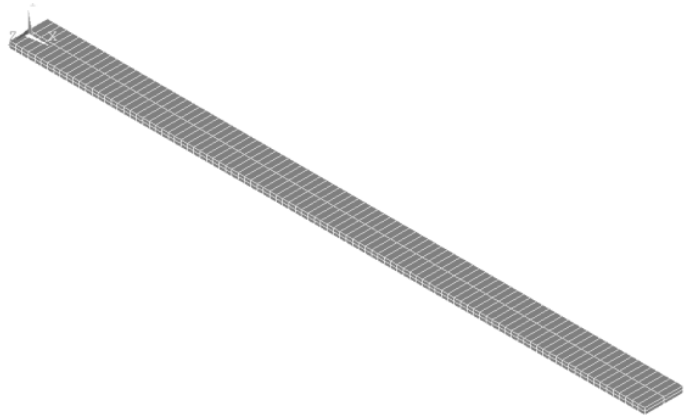

Fig. 7. FEM model of cantilever beam.

Table 2. Natural frequencies of each mode

\begin{tabular}{|c|c|c|c|c|c|c|}
\hline \multirow{2}{*}{ Mode } & \multicolumn{2}{|c|}{ Set number $1(\mathrm{~Hz})$} & \multicolumn{2}{|c|}{ Set number $2(\mathrm{~Hz})$} & \multicolumn{2}{|c|}{ Set number $3(\mathrm{~Hz})$} \\
\hline & Theory & FEM & Theory & FEM & Theory & FEM \\
\hline 1 & 8 & 8.1 & 9 & 8.9 & 10 & 9.7 \\
\hline 2 & 51 & 50.8 & 56 & 55.9 & 61 & 61 \\
\hline 3 & 142 & 142.3 & 157 & 156.5 & 171 & 170.7 \\
\hline
\end{tabular}




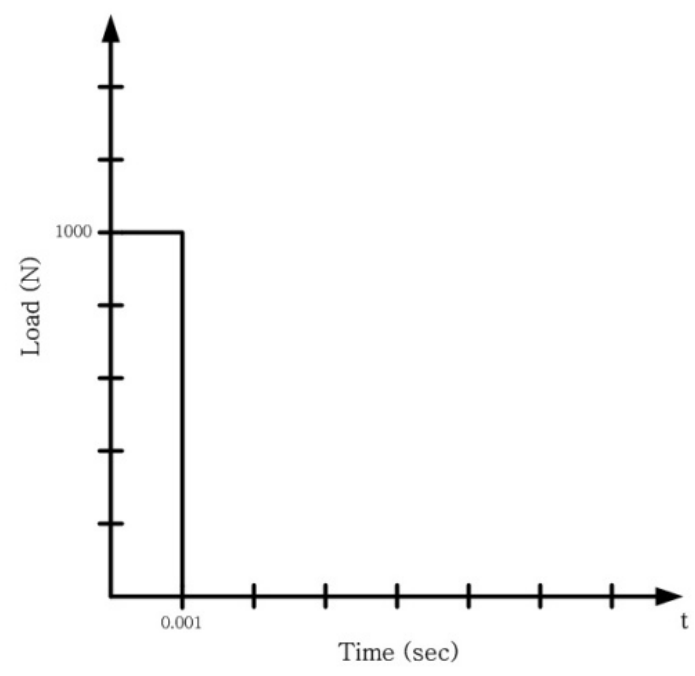

Fig. 8. Mode shapes and natural frequencies for the first three modes.

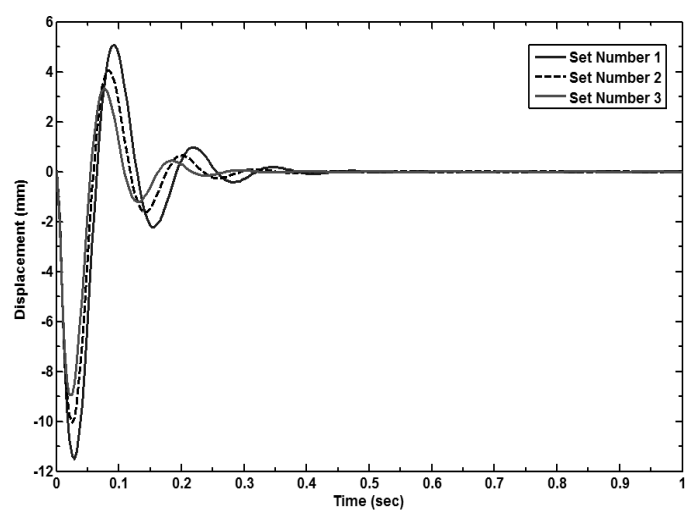

Fig. 9. Tip displacement for three set number for damping $1 \%$ of critical.

in Table 1. So far, it cannot be decided which dimension is best suitable for design. Therefore, further results are evaluated based on harmonic analysis.

\subsection{Harmonic analysis}

In order to strengthen the claim made in the modal analysis, harmonic analysis was also performed. The "Full" method was used for this structure, because it is simpler and easier to use as compared to the "Reduced" and "Superposition" methods. However, this method makes use of full stiffness and mass matrices,

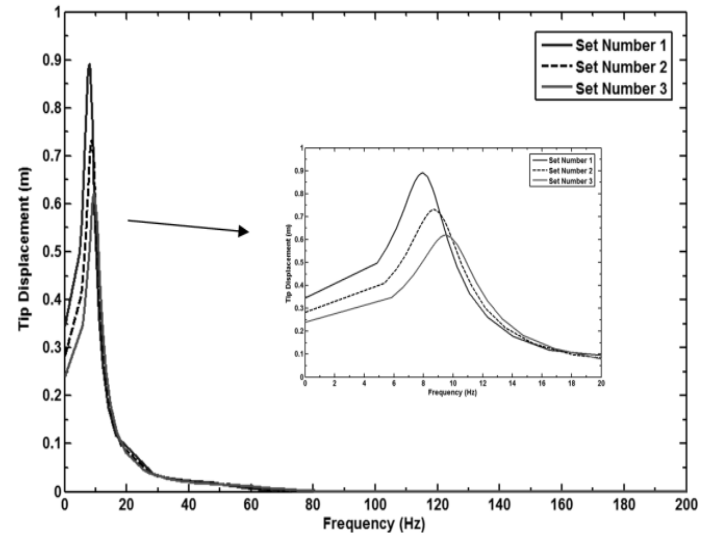

Fig. 10. Tip displacement harmonic response.

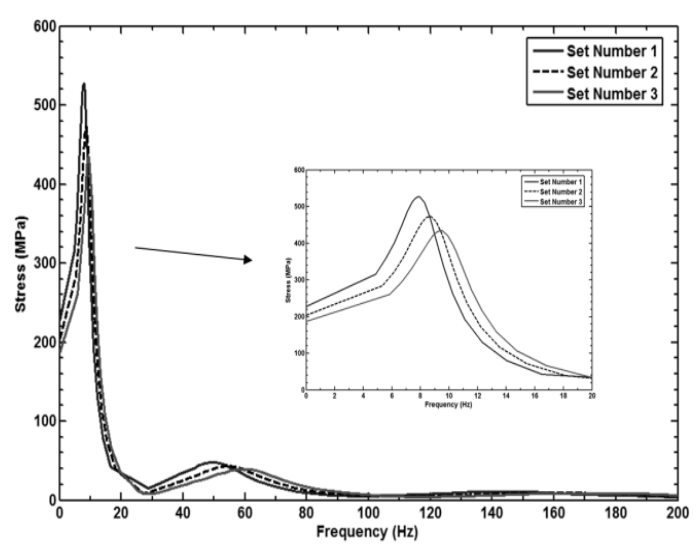

Fig. 11. Working bending stress at fixed end.

and thus it is also a slower and costlier option[3,4]. For harmonic analysis we applied the dynamic load on the beam varying the frequency from $1 \mathrm{~Hz}$ to $200 \mathrm{~Hz}$ of amplitude $1000 \mathrm{~N}$. In this analysis, we will compare the value of stresses for all three cases rather to limit them at $100 \mathrm{MPa}$ which was defined as maximum for static load condition since we are dealing with dynamic load.

At their first resonance frequency, the tip displacements for set number 1, 2 and 3 were compared as shown in Fig. 10. An exaggerated view is also presented of first mode resonance.

Then the bending stress due to dynamic load was evaluated as shown in Fig. 11. The dimension obtained from FEM analysis under goes the minimum bending stress at fixed end. 


\section{CONCLUSIONS}

The objective of this paper was to obtain the optimum dimensions of a cantilever beam which has minimum weight as well as minimum vibration. The FEM program such as ANSYS gives us the optimum results for dimensions having minimum volume, but not the exact width and height which have minimum vibration. So the modal and harmonic analyses have been presented. We have found that depending on excitation frequency, the best design may differ due to resonance effects. In this case, the dimension obtained from FEM analysis qualifies the design for static as well as dynamic loading conditions. It is concluded that modal and harmonic analyses are indispensable for optimum safe design of a structure, in addition to static analysis. This concept, with aid from FEM commercial code, can be used for more efficient, safe, and light structure design which can save the energy.

\section{REFERENCES}

[1] Brown, J. M., Three Dimensional Shape Optimization with Probabilistic Constraints using Parametric Session File \#0699, Air Force Research Laboratory, Wright Patterson, Air Force Base(AFB)

[2] Shunk, D. L., Optimization of Manufacturing
System Design, North Holland, (1990)

[3] Lilly, J., Fyfe, K., Jones, S., Mckay, B., Muradali, A., Sorensen, C., Skoczylas, P., Toogood, R., and Johns, K., ANSYS Tutorials, University of Alberta, Alberta, (2002)

[4] Herrin. D., Modal and Hamonic Analysis using ANSYS, University of Kentucky, Kentucky, USA, (2004)

[5] Brown, D. L., Allemang, R. J., and Halvorsen, W. G., "Impulse technique for structural frequency response testing", J. of the Acoustical Society of America, 63, 1-81, (1978)

[6] L. Wang, R. V. Grandhi and D. Hopkins, "Structural reliability optimization using an efficient safety index calculation procedure", Int. J. Numer. Meth. Engng., 38, 1721 - 1738, (1995)

[7] Vudathu, S. P., Duganapalli, K. K., Laur, R., and Kubalinska, D., "Yield analysis via induction of process statistics into the design of MEMS and other Microsystems", Microsystem Technologies, 13, 1545-1551, (2007)

[8] Cholaseuk, D., "A stress-base material distribution method for optimum shape design of mechanical parts", Thammasat International J. of Science and Technology, 11, 17-21, (2006)

[9] Cibson, R. F., Principles of Composite Material Mechanics, 2nd ed., CRC Press, New York, (2007) 